\title{
Mechanical reliability of dental grade zirconia after laser patterning
}

\author{
E. Roitero ${ }^{1,2,3}$, M. Anglada ${ }^{1,2}$, F. Mücklich ${ }^{3}$ and E. Jiménez-Piqué ${ }^{1,2, *}$ \\ ${ }^{1}$ Department of Materials Science and Metallurgical Engineering, Universitat Politècnica de \\ Catalunya, Campus Diagonal Besòs - EEBE, Barcelona 08019, Spain. \\ ${ }^{2}$ Centre for Research in Multiscale Engineering of Barcelona, Universitat Politècnica de \\ Catalunya, Campus Diagonal Besòs - EEBE, Barcelona 08019, Spain. \\ ${ }^{3}$ Department of Materials Science and Engineering, Saarland University, Saarbrucken 66123, \\ Germany. \\ *Corresponding Author \\ e-mail: emilio.jimenez@upc.edu
}

\section{Abstract}

The aim of this work is to test the mechanical properties of dental zirconia surfaces patterned with Nd:YAG laser interference ( $\lambda=532 \mathrm{~nm}$ and $10 \mathrm{~ns}$ pulse). The laser treatment produces an alteration of the topography, engraving a periodic striped pattern. Laser-material interaction results mainly in thermal effects producing microcracking, phase transformation and texturization. The role of such microstructural modifications and collateral damage on the integrity and mechanical performances has been assessed.

Laser patterned discs of zirconia doped with $3 \%$ mol yttria (3Y-TZP) have been tested before and after a thermal treatment to anneal residual stresses and revert phase transformation. Both groups of samples behave in a similar manner, excluding residual stresses and phase transformation from the origin of properties modification

Results show that laser patterning induces a minor decrease in mechanical properties and surface integrity of 3Y-TZP surfaces. The biaxial strength decreases as a consequence of the damage induced by laser patterning. Fractographic observations identify preexisting defects enlarged by local laser interaction as the fracture origins. The Hardness and Young modulus of treated surfaces tested with nanoindentation also decrease slightly after laser treatment and this may be attributed to laser-induced microcracking.

Keywords: laser treatment, 3Y-TZP, direct laser interference patterning, mechanical properties. 


\section{Introduction}

Tetragonal Zirconia Polycrystal stabilized with 3 mol. \% $\mathrm{Y}_{2} \mathrm{O}_{3}$ (3Y-TZP) has been progressively employed in restorative dentistry thanks to the excellent combination of biocompatibility, mechanical performance and aesthetics [1]. The good strength and toughness among available bioceramics make it suitable for structural applications as crowns, bridges, abutments and more recently implants [2]. The main drawback for biomedical application is the lack of long-term stability caused by Low Temperature Degradation (LTD). In humid environments this family of materials is susceptible to spontaneous transformation from the metastable tetragonal to the monoclinic phase. The resulting volume increment causes microcracking and grains pull-out, with a consequent loss of surface integrity and mechanical properties[3].

In order to improve the biological response or the mechanical adhesion to other materials (enamels or dental cement and resin) different surface treatments have been proposed to modify the topography and roughness of these restorations. Each application may require a different kind of topography in terms of geometry, regularity and scale-size (generally, the desired features are in the micro- and nano-scale [4]). Periodical and defined micro-patterns with features smaller than $100 \mu \mathrm{m}$ can be produced by mechanical micromachining, stamp transfer molding and laser-based techniques [5]. Laser-based methods are faster, contact-less, more accurate in pattern reproduction and are relatively easy to implement $[6,7]$.

Direct Laser Interference Patterning (DLIP) offers a fast and accurate alternative to introduce controlled topography at the micrometric and sub-micrometric scale [6]. Laser beams are guided through the optical setup to interfere on the surface to be treated generating a periodical intensity distribution. Different geometries can be produced varying the number of beams and the optical setup (the simplest are lines and dots) [8]. The incoming laser pulse locally melts the substrate and capillary forces generated by temperature gradient cause material flow and pattern formation [9]. Very steep thermal gradients arise during the laser pulse, as a consequence of the low thermal conductivity of 3Y-TZP [10]. The resulting thermal shock produces recrystallization in form of columnar grains and intergranular microcracking, down to $1 \mu \mathrm{m}$ depth. The high thermal load induces also $t \rightarrow m$ phase transformation, texturization of $t$ phase and residual stresses and strains. All these modifications are homogeneously distributed along the topography and affect the first micrometer of material below the treated surface. Further details about type and distribution of collateral damages after laser treatment can be found in previous work of some of the authors [11,12].

Understanding the effect of such microstructural changes induced by DLIP on the mechanical properties of 3Y-TZP is important in order to ensure material reliability. Since in ceramic materials fracture is governed by critical defects [13], it is necessary to understand how the laser treatment could affect defect population and distribution, especially on the surface. At date, few studies [14-16] about the mechanical properties of laser patterned zirconia have been reported and mainly involved hardness measurements alone [15,16]. Only C. Daniel et al. [14] have tested uniaxial flexural strength of dental grade zirconia patterned with DLIP: they reported a notable increment in flexural strength attributed to the nanometric size grains and compressive residual stresses caused by laser treatment. However, it is not possible to 
generalize such observations since laser treatments encompass a wide variety of effects of laser interaction with matter resulting in very different microstructural changes [11].

Therefore, the objective of this study is to understand the changes produced in mechanical reliability by laser patterning. For that, biaxial flexural strength is performed on laser treated samples with a subsequent fractographic analysis to identify the critical defects. Surface hardness and Young modulus are tested with nanonindentation in order to evaluate the integrity of the laser affected layer. The laser induced damage is correlated with variations in mechanical response

\section{Materials and method}

\subsection{Material processing}

Commercially available powder of Tetragonal Polycrystalline Zirconia stabilized with 3\% molar $\mathrm{Y}_{2} \mathrm{O}_{3}$ (TZ-3YSB-E, Tosoh, Tokyo, Japan) was cold-isostatic pressed at $200 \mathrm{MPa}$ and then sintered at $1450^{\circ} \mathrm{C}$ for two hours $\left(3^{\circ} \mathrm{C} / \mathrm{min}\right.$ heating rate). The rods were cut into discs of approximately $9 \mathrm{~mm}$ diameter and $2 \mathrm{~mm}$ thickness. The surface of the samples was ground and polished with diamond suspensions of $30-6-3 \mu \mathrm{m}$ particle size with a final step of colloidal silica. The measured final density was $6.03 \pm 0.02 \mathrm{~g} / \mathrm{cm}^{3}(99.67 \%$ of theoretical density) with a grain size of $0.31 \pm 0.08 \mu \mathrm{m}$ (intercept distance). The obtained material has biomedical grade, according to ISO 13356:2013 [1]. These samples were then split into two groups: the first group did not undergo any further modification while the other discs were laser patterned with DLIP. The samples that did not undergo any further treatment were labelled Not Treated (NT) and served as reference material. The discs that were laser-treated were labelled Laser Patterned (LP) and were further divided into two groups: one group (LP) did not undergo any further treatment after patterning while the other discs (LP+TT) were annealed after the laser treatment. The annealing treatment was performed in an air furnace at $1200^{\circ} \mathrm{C}$ during 1 hour, with the purpose of eliminating residual stresses and revert the monoclinic phase to tetragonal (further details about the effect of the thermal treatment on LP samples can be found in [12]).

\subsection{Laser patterning}

A Q-switched Nd:YAG laser (Spectra Physics Quanta-Ray PRO210) with a fundamental wavelength of $1064 \mathrm{~nm}$ was employed in the DLIP setup. The output wavelength of $532 \mathrm{~nm}$ obtained by second harmonic generation was used for patterning of zirconia discs surface. The repetition rate and the pulse duration of the laser were $10 \mathrm{~Hz}$ and $10 \mathrm{~ns}$, respectively. All samples were treated with one single pulse and with a fluence of $4 \mathrm{~J} / \mathrm{cm}^{2}$. An optical setup with two interfering beams allows producing a striped pattern consisting of alternating valleys and peaks with a peak-to-peak distance (i.e. periodicity) of $10 \mu \mathrm{m}$. The two-beam interference results in a plane sinusoidal intensity distribution $I(x, y)$ on the surface of the sample, as schematized in [9]. It can be described by:

$$
I(x, y)=I_{o}\left[\cos \left(\frac{4 \pi x}{\lambda} \sin \alpha\right)+1\right]
$$


where $I_{o}$ is the intensity of the laser beam before splitting, $\lambda$ is the laser wavelength and $\alpha$ is the half angle between the interfering beams. Further details about the technique and the setup employed can be found in [17] and [18].

\subsection{Biaxial bending and fractography}

The biaxial strength of NT, LP and LP + TT discs was tested with the ball on three balls (B3B) method [19]. Fracture load was measured on discs subjected to biaxial flexure with a testing fixture with cobalt-cemented tungsten carbide balls of $5.9 \mathrm{~mm}$ diameter and a loading rate of $200 \mathrm{~N} / \mathrm{s}$, using a servo-hydraulic testing machine (8511, Instron). Then, the biaxial strength was calculated according to the numerical approximation proposed by Börger et al. in [20]:

$$
\sigma_{f}=f\left(\frac{t}{R}, \frac{R_{a}}{R}, v\right) \cdot \frac{F}{t^{2}}
$$

where $F$ is the fracture load, $t$ is the sample thickness and $f$ is a dimensionless parameters depending on the geometry of the disc (the radius of the disc $R$, the support radius $R_{a}=3.4 \mathrm{~mm}$ and the Poisson's ratio $v \approx 0.3$ for 3Y-TZP, see [20] for further details).

A statistical analysis of the results was performed applying the conventional Weibull theory $[21,22]$. The distribution function is defined as:

$$
P\left(\sigma_{f}\right)=1-e^{-\left(\frac{\sigma_{f}}{\sigma_{0}}\right)^{m}}
$$

where $P$ is the cumulative probability of failure, $\sigma_{f}$ is the biaxial strength, $\sigma_{0}$ is the Weibull characteristic strength, and $m$ is the Weibull modulus.

For the graphical evaluation of $m$ and $\sigma_{0}$ (liner regression) the measured strength data were ranked in increasing order and numbered from 1 to $\mathrm{N}$. Then, the single strength values $\sigma_{f i}$ were related to the failure probability $P_{i}$ according to the following relation:

$$
P_{i}=\frac{1-0.5}{N}
$$

where $i$ is the ranking number and $\mathrm{N}$ is the total number of measurements.

Observation of the fracture surfaces and identification of fracture origin was performed with Scanning Electron Microscopy (SEM) (Neon 40, Carl Zeiss).

\subsection{Nanoindentation and scratch test}

The integrity and mechanical properties of treated surfaces were assessed with nanoindentation. Tests were performed using a MTS Nanoindenter XP with a continuous stiffness measurement module and with a Berkovich diamond tip calibrated against fused silica standard. Results were analyzed with the Oliver and Pharr method [23].

Scratch test were performed with a CMS Revetest using a Rockwell C diamond tip, with increasing load from $0 \mathrm{~N}$ to $60 \mathrm{~N}$ with a scratch length of $2 \mathrm{~mm}$. 


\section{RESULTS}

\subsection{Strength analysis}

The mean biaxial flexural strength results are shown in Table 1. Both LP and LP+TT samples show a slight decrease in strength compared to NT samples. There is no significant difference between the strength of laser patterned samples before (LP) and after (LP+TT) the thermal treatment.

\begin{tabular}{lccc} 
& $\begin{array}{c}\boldsymbol{\sigma}_{f} \\
{[\mathrm{MPa}]}\end{array}$ & $\begin{array}{c}\boldsymbol{\sigma}_{o} \\
{[\mathrm{MPa}]}\end{array}$ & $\boldsymbol{m}$ \\
\hline $\begin{array}{l}\text { Not Treated } \\
\text { (NT) }\end{array}$ & $1347 \pm 64$ & 1400 & $7.8 \pm 0.6$ \\
$\begin{array}{l}\text { Laser Patterned } \\
\text { (LP) }\end{array}$ & $1190 \pm 81$ & 1228 & $16.4 \pm 1.1$ \\
$\begin{array}{l}\text { Laser Patterned + Thermal Treatment } \\
\text { (LP+TT) }\end{array}$ & $1161 \pm 112$ & 1199 & $16.0 \pm 0.7$
\end{tabular}

Table 1. Mean biaxial strength $\left(\sigma_{f}\right)$, Weibull characteristic strength $\left(\sigma_{0}\right)$ and Weibull modulus $(m)$ for NT, LP and LP+TT samples.

Weibull analysis (Fig.1, Table 1) confirmed the decrease of strength associated to laser patterning. The distribution of the results of LP and LP+TT samples is not only shifted towards lower strength values but it is also less scattered, if compared to NT samples. This is evidenced in the increase of the Weibull modulus (Table 1). Again, the thermal treatment after laser patterning has no substantial influence.
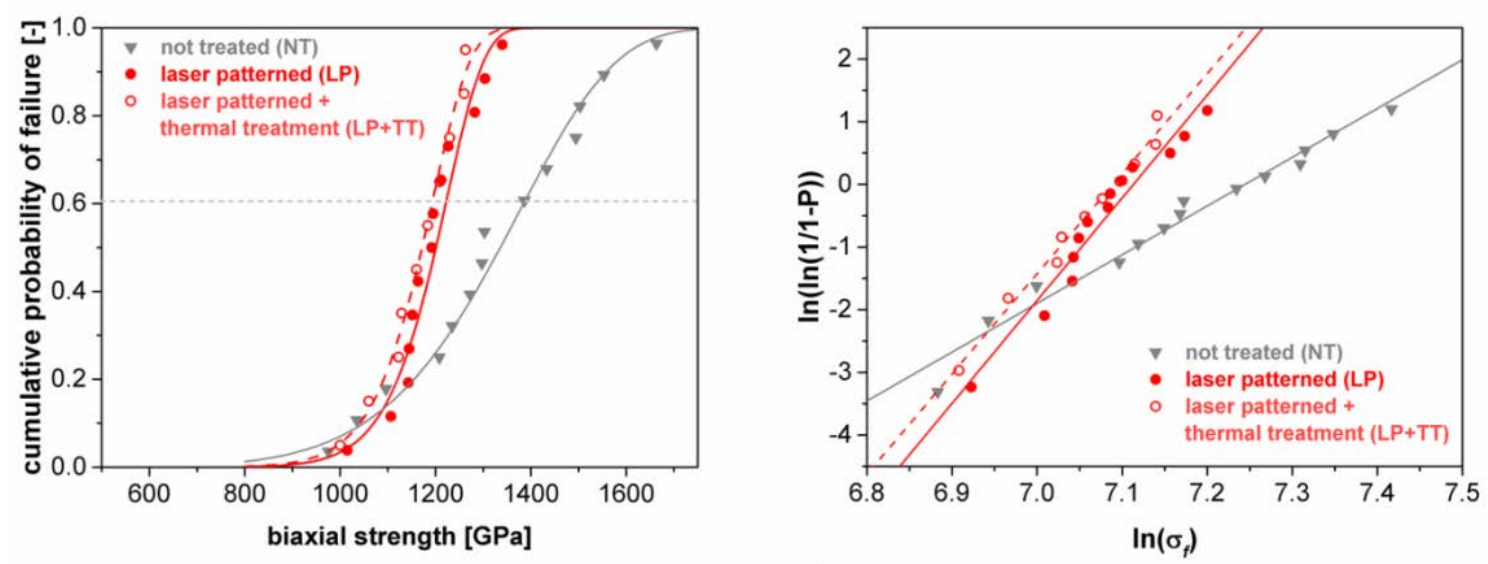

Fig. 1: Weibull distributions of not treated (NT, grey triangles) and laser patterned before (LP, hollow red circles) and after thermal treatment ( $L P+T T$, red circles). (A) Linear scale and (B) logarithmic scale graph.

Because of the limited number of samples, interpretations regarding the Weibull distribution have to be subjected to caution. It seems, however, that laser patterning plays some role on the critical defect size and distribution. 


\subsection{Fractography}

In all tested samples, fracture initiated at the center of the disc on the surface undergoing biaxial tension, as expected since this is the volume of the disc experiencing the highest tensile stresses [20]. Identification of fracture origins in LP and LP+TT samples was possible because a mirror zone was present on all examined discs (Fig. 2A, B). However, it was not always possible to identify univocally the critical defect (Fig. 3B).
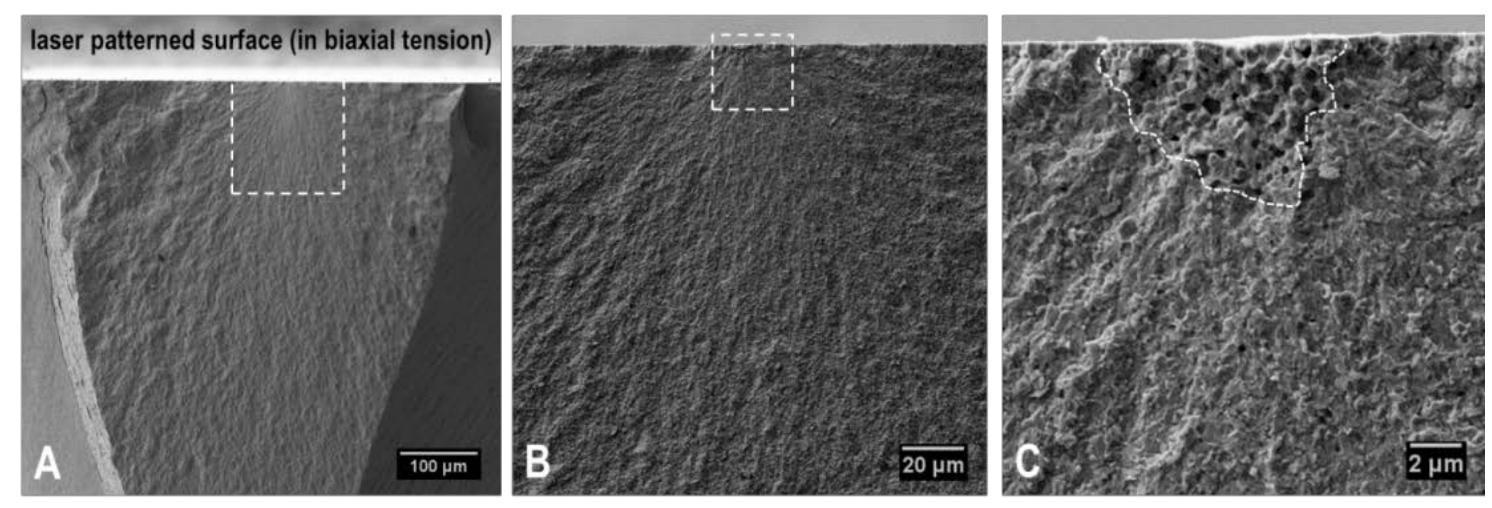

Fig. 2: FESEM images of the fracture surface of a $L P$ sample $\left(\sigma_{f}=1143 \mathrm{MPa}\right)$. (A) A mirror zone can be identified as the origin of fracture on the laser treated surface undergoing biaxial tension in the central area of the disc (maximum stress). In (B), the magnification of the mirror zone region allows to identify the fracture origin as a defect close to the laser treated surface. In (C), the critical defect responsible of fracture can be identified as porous

The identification of critical defects was possible both in LP and LP+TT samples: processing defects like porosity (Fig. 3A, C, D) or low density regions (Fig. 2 C) were found to be at the origin of fracture for both group of samples. Also in this case no substantial difference was observed between LP and LP+TT samples. 


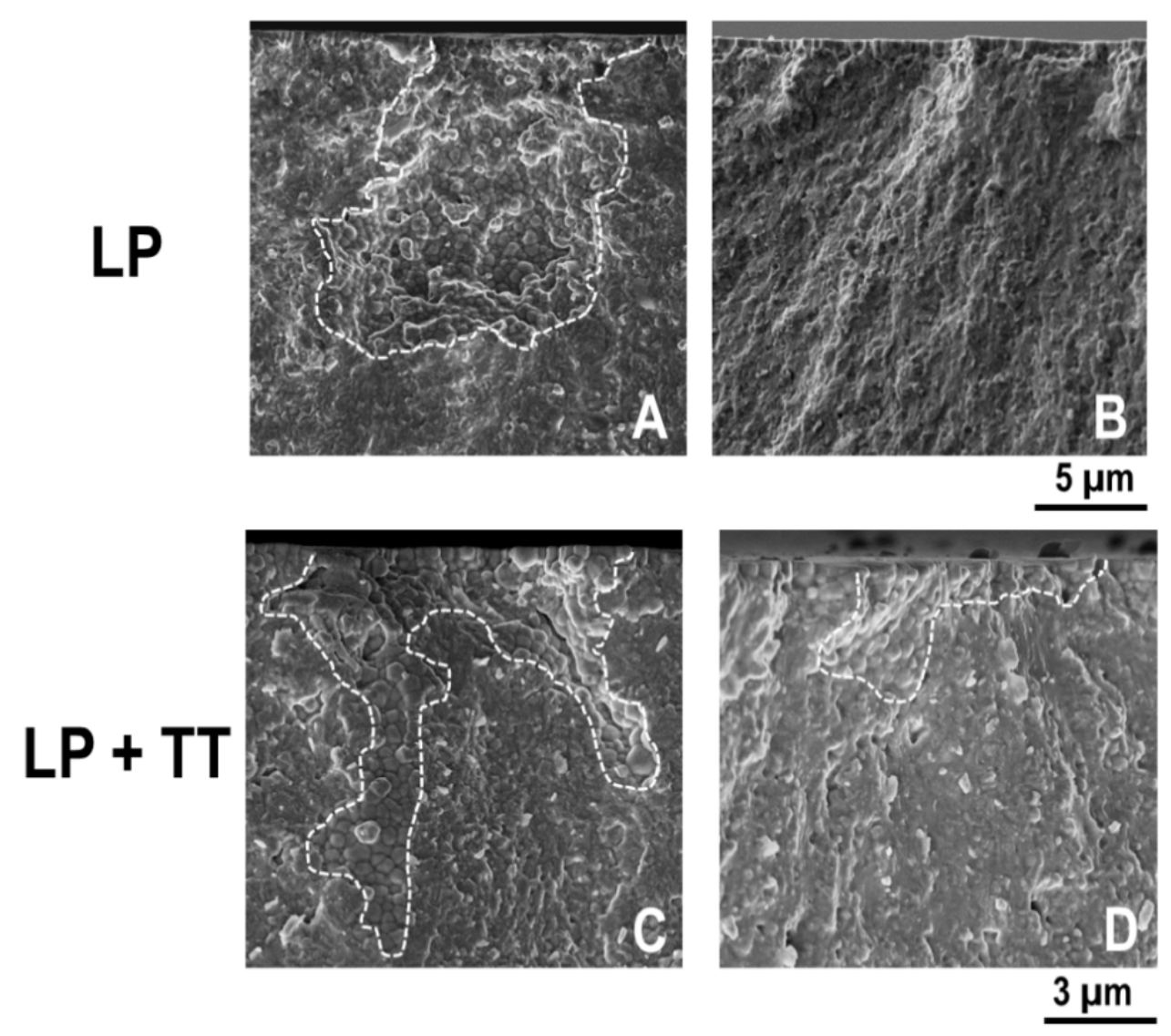

Fig. 3: FESEM images of critical defects at the origin of fracture of $L P(A, B)$ and $L P+T T$ samples $(C, D)$. Biaxial strength of each samples is $1015 \mathrm{MPa}(A), 1304 \mathrm{MPa}(B), 1128 \mathrm{MPa}(C)$ and $1263 \mathrm{MPa}(D)$, respectively. In (B) the critical defect could not be identified.

The fracture in the mirror region close to the laser treated surface is transgranular, both in LP and LP+TT samples (see Fig. 4A). With a closer look (Fig. 4B), it is possible to distinguish a thin layer of intergranular fracture $(\approx 1 \mu \mathrm{m}$ thick) corresponding to the first layer of grains below the treated surface. 


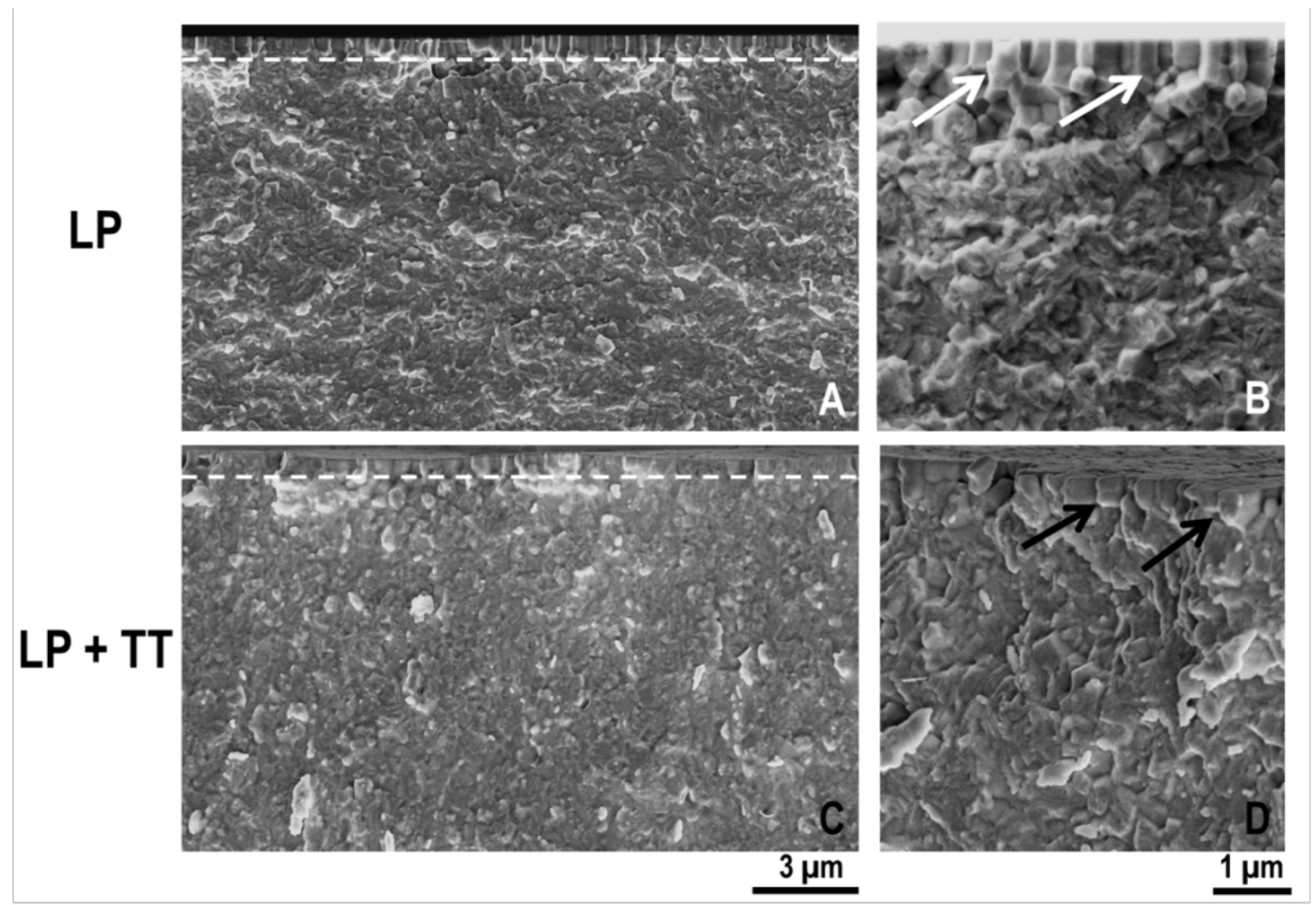

Fig.4. FESEM images of fracture surfaces of $L P(A, B)$ and $L P+T T(C, D)$ discs. In the higher magnification images $(B, D)$ the distinction between the intergranular fracture layer (black and white arrows) from the transgranular fracture layer is more evident.

The intergranular fracture region corresponds to the thermally affected volume during laser treatment [11]. In this volume, fracture propagates along the pre-existing microcracks net on the surface (Fig 4B, D). Comparing LP to LP+TT fracture surfaces, it is interesting to note that the aspect of the grains in the intergranular fracture region is different: in LP samples, the corners of columnar grains are sharp while in LP+TT samples they are rounded and less defined.

\subsection{Nanoindentation and scratch test}

The integrity of laser treated surfaces was assessed with nanoindentation tests, evaluating the evolution of Hardness and Young Modulus as a function of indenter penetration. Laser patterned samples (LP and LP+TT) were compared to not treated 3Y-TZP (NT). Results are reported in Fig. 5.
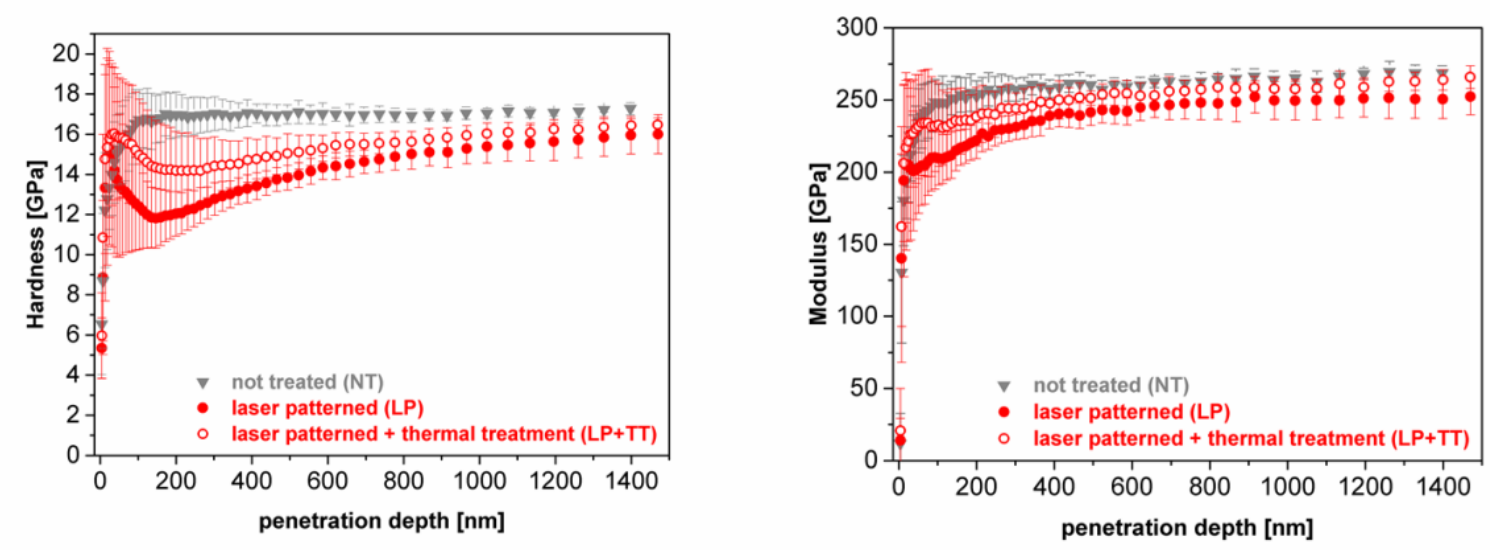
Fig. 5. Nanoindentation tests results of not treated (NT, grey triangles) and laser patterned before (LP, hollow red circles) and after thermal treatment ( $L P+T T$, red circles) samples. In $(A)$ the Hardness and in $(B)$ the Young Modulus are plotted versus the indenter penetration depth.

For the not treated material a constant value of $17 \mathrm{GPa}$ of Hardness and $250 \mathrm{GPa}$ of Young Modulus can be defined after $150 \mathrm{~nm}$ of indenter penetration, compatibly with values reported typically in the literature for 3Y-TZP [24]. LP and LP+TT samples show a slight decrease both in Hardness and Young Modulus, which are recovered as the indenter penetrates further in depth of the laser treated surfaces. This loss can be related to the microcracked surface and the altered microstructure (elongated grains and $t \rightarrow m$ phase transformation) due to thermal effects caused by laser treatment $[25,26]$ as well as the induced topography of the sample. No substantial difference can be observed between LP and LP+TT samples.

The scratch test performed provide a qualitative evaluation of the resistance to shear and friction of the laser patterned samples compared to a not treated surface of 3Y-TZP.

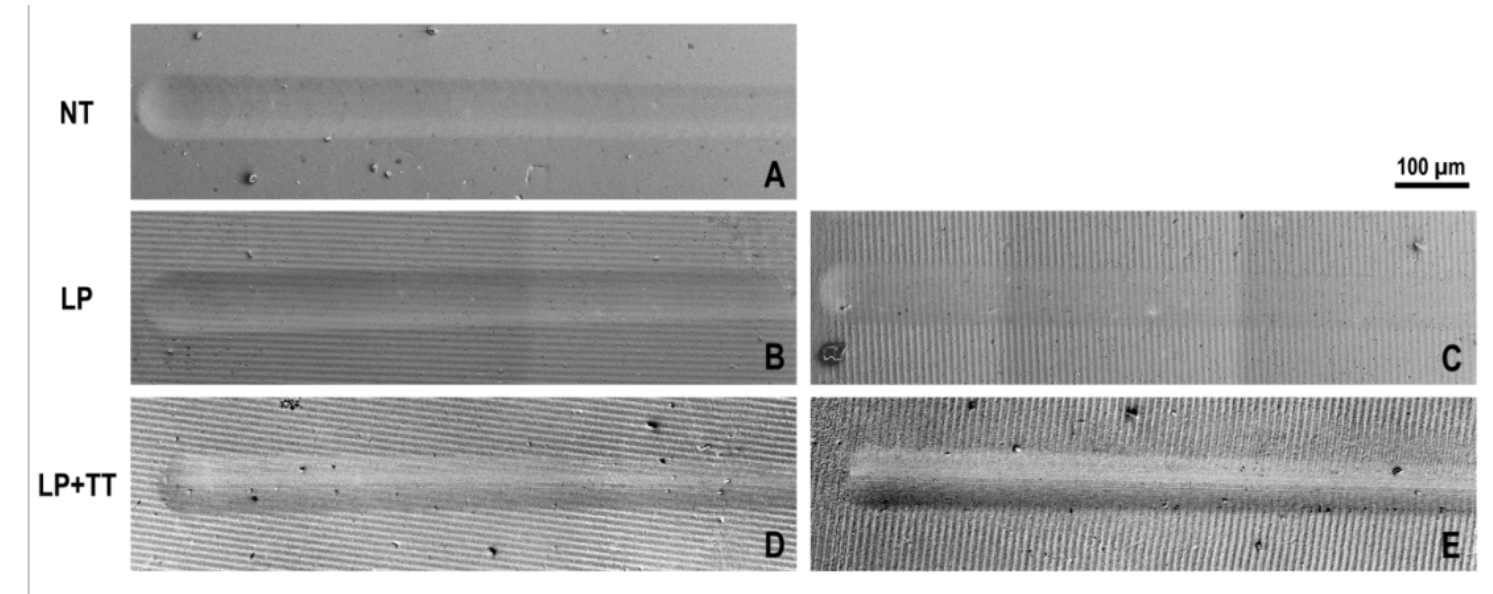

Fig.6. FESEM images of the scratches on not treated $(A)$ and laser patterned before $(B, C)$ and after $(D, E)$ the thermal treatment. The relative orientation of pattern stripes and scratch direction is parallel in $B$ and $D$ and orthogonal in $C$ and $E$.

In Fig.6 it is possible to observe that the scratches have similar sizes for all kind of samples. For the chosen parameters no fracture and material spallation occur, only a permanent plastic deformation in the wake below the indenter tip. No substantial difference can be observed between not treated (NT) and laser patterned samples before (LP) and after (LP+TT) thermal treatment, a part from the presence of cone cracks in the scratch on NT samples only. It is interesting to observe that the striped topography produced by laser is still appreciable inside the scratch wake. Furthermore, the direction of the scratch relative to pattern lines orientation seems not to be significant, showing an isotropic behavior.

\section{Discussion}

From the obtained results, laser patterning seems to be at the origin of a slight decrease in the mechanical properties of 3Y-TZP. On the other hand, the thermal treatment seems not to affect the mechanical properties of the laser patterned samples. The biaxial strength of LP and $\mathrm{LP}+\mathrm{TT}$ samples measured with B3B method is lowered while the Weibull modulus in increased (see Table 1 and Fig. 1). These features in the distribution are due to a change in the critical defects population, since the distribution for LP and LP+TT samples is less scattered and shifted 
towards lower values of $\sigma_{f}$ when compared to not treated 3Y-TZP (Fig. 1). In the following paragraph we will discuss how the laser treatment may affect defect population.

In a previous work [11] it has been shown how laser patterning with ns-pulsed laser affects the microstructure of 3Y-TZP. Main modifications are concentrated in the first $\mu \mathrm{m}$ below the treated surface and have thermal origin. Microcracking, recrystallization, $t \rightarrow m$ phase transformation, $t$-phase texturization and residual stresses are the main consequences of the thermal shock produced during laser treatment. The presence of $m$-phase and residual stresses should not be responsible of the decrease in mechanical properties, since there is no difference in the biaxial strength and Weibull modulus in laser treated samples before (LP) and after the thermal treatment $(\mathrm{LP}+T \mathrm{TT})$. In fact, annealing at $1200^{\circ} \mathrm{C}$ for $1 \mathrm{~h}$ is able to relief residual stresses and revert $m$-phase to $t$-phase [12]. The relatively small number of samples does not allow to conclusively discern a difference between the LP and LP+TT samples, however, the small difference in values may indicate that residual stresses are very shallow and do not contribute to significant changes in strength.

The microcracked surface layer composed of columnar grains (maximum thickness of 1 $\mu \mathrm{m})$ [11] corresponds to the intergranular fracture layer observed in the fractographic images (see Fig. 3). To understand if the decrease of strength is caused by this affected layer of material, a simple model is proposed. The microcracked surface layer is modeled as a straight through edge crack of thickness $a$. Then, the stress intensity factor $K_{/}$for the applied stress $\sigma_{a p p}$ would be:

$$
K_{I}=Y \sigma_{a p p} \sqrt{\pi a}
$$

The geometric factor $Y$ is 1.12 for this defect geometry [27]. Knowledge of the fracture toughness of the material is needed to determine $a$. For 3Y-TZP the values reported in the literature are scattered, depending on the method employed for its determination, because of the transformation toughening phenomenon and the nanometric grain size of 3Y-TZP. In this study we employed a value of $K_{I C}=4.1 \mathrm{MPa} \mathrm{m}^{1 / 2}$ [28]. Considering $\sigma_{a p p} L P=\sigma_{0 L P}=1228 \mathrm{MPa}$ and $\sigma_{\text {app LP+TT }}=\sigma_{0 L P+T T}=1199 \mathrm{MPa}$, the thickness of the affected layer acting as critical defect then should be $a_{L P}=2.8 \mu \mathrm{m}$ and $a_{L P+T T}=3.0 \mu \mathrm{m}$ for laser patterned samples before and after the thermal treatment, respectively. Since the laser affected layer reaches a maximum depth of 1 $\mu \mathrm{m}$, the damage produced by laser alone cannot be at the origin of the fractures. This is in accordance with fractographic observations (Fig. 3): all the identified critical defects are porosities and low-density regions that most probably are produced during processing and sintering steps. If this were the case, the critical defect can be modeled as circular surface crack of radius $a$. Then, the geometric factor $Y$ would become 0.9 and the radius of the circular critical defect calculated with (5) would be $a_{L P}=4.4 \mu \mathrm{m}$ and $a_{L P+T T}=4.7 \mu \mathrm{m}$, respectively. These values are in accordance with the experimental observations and the critical defects identified (Fig $2 \mathrm{C}$ and Fig. 3).

This kind of processing defects close to the surface have been identified as the most conventional cause of failure in 3Y-TZP [29]. However, there have to be some kind of influence of the laser treatment on the defect population that causes the observed change in Weibull distribution of failures (Fig. 1). Looking in more detail at some of the critical defects observed 
during fractography of a LP sample, it is possible to observe that the pre-existing defect (like a pore) may have been modified by the laser treatment.

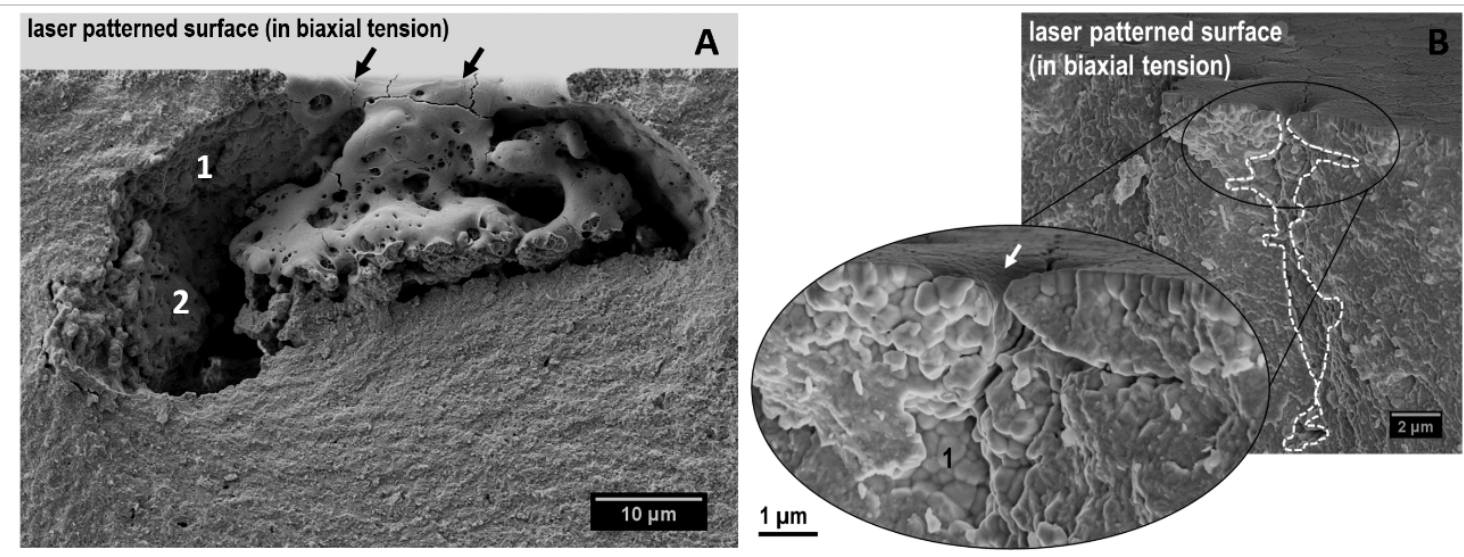

Fig. 7. FESEM magnification of critical defects showing the interaction of laser melting with pre-existing defects of the material. (A) LP sample ( $\sigma_{f}=869 \mathrm{MPa}$ ) with a big pore on the surface: the inner walls of the pore have rounded grains (1) typical of sintering step but also show molten and resolidified material (2) typical of laser-induced melting. There is a molten rim on the laser treated surface (indicated by black arrows). (B) $L P+T T$ sample $\left(\sigma_{f}=1208 \mathrm{MPa}\right.$ ) with an elongated pore on the surface: the inner walls of the pore have rounded grains (1) typical of sintering step and there is a molten rim (indicated by white arrow) opening the pore to the surface.

Examples of defect-laser interaction can be seen in fig. 7. Molten and resolidified material can be seen inside a pore in a LP sample (fig. 7A). There is a molten rim on the lasertreated surface (indicated by black arrows) and some partially molten material in the middle of the pore, as if the top of the defect has collapsed due to material melting. Similar observation can be done on a LP+TT sample (Fig. 7B): the elongated pore is opened to the surface by material melting (see again the presence of a molten rim indicated by the white arrow). Therefore it is possible that material melting on the surface caused by the laser pulse may interact with defects already present inside the material (on the surface or right below it). Laser induced melting would result in the enlargement of preexisting defects. This would be compatible with the observed reduction in the Weibull characteristic strength observed after laser treatment (Table 1): the circular critical defect for not treated 3Y-TZP is $3.4 \mu \mathrm{m}$ and becomes $4.4 \mu \mathrm{m}$ after laser patterning. This effect could also be more pronounced in terms of strength reduction for defects very close to the surface that after laser treatment would become open to the surface, while melting of pre-existing open porosity may not have a significant effect.

It has been observed that in dielectrics the presence of preexisting defects on the surface (for instance absorbing impurities, cracks or rough cavities) could modify the normal laser absorption characteristics of the surface [30,31]. In fact, those defects can enhance laser absorption and therefore local material melting. Furthermore, if the defect has a lower heat transmission coefficient than the matrix (a pore filled with air in zirconia), the defect could also act as a thermal barrier causing local overheating [32]. These observations may be valid for $3 Y$ TZP and corroborate the hypothesis of defect enlargement due to material melting during laser exposure.

Nanoindentation results show that the Hardness and Young modulus of the surfaces of 3Y-TZP decrease after the laser patterning (Fig. 5). The decrease in properties remains the same after the thermal treatment. Likewise discussed in previous paragraph about mechanical properties, microcracking should be at the origin of this decrease in surface properties. 
On the other hand, the laser affected layer seems not to strongly affect the integrity of the surface upon scratch (Fig. 6). In LP and LP+TT samples the damage produced by the spherical indenter does not compromise surface integrity more than in NT samples. Cone cracks are appreciated in the NT sample, typical of a brittle material [33]. This type of cracking cannot be appreciated in the LT materials. This can be attributed to the fact that the surface microcracks accommodate the deformation at the surface of the sliding contact, hindering, consequently, the formation of such cone cracks.

\section{Conclusions}

Laser patterning with DLIP induces a minor decrease in mechanical properties and surface integrity of 3Y-TZP. The biaxial strength (B3B) and the Hardness and Young modulus of the treated surfaces decrease as a consequence of the damage induced by laser patterning. Laser-affected layer could not be at the origin of fractures alone since its thickness is only $1 \mu \mathrm{m}$, well below the critical defect size. Laser interaction with pre-existing defects close to the surface result in an enlargement of such surface defects, thanks to focalization of laser radiation and subsequent material melting and damage. We believe that those enlarged critical defects are at the origin of the decrease in Weibull characteristic strength and the increase in Weibull modulus, as supported by the calculations of the critical defect size.

A thermal treatment after laser patterning does not alter further surface integrity and mechanical properties of laser patterned 3Y-TZP.

Laser treatment does not result in a significant loss of mechanical properties. This make this technique suitable to pattern surfaces for dental applications. Nevertheless, change in Weibull strength and scattering has to be taken into account to assure

\section{Acknowledgements}

Work supported in part by the Ministry of Economy and Competitiveness (MINECO) of Spain (project ref. MAT2014-60720-R), the CREATE-Network Project, Horizon 2020 Program of the European Commission (RISE Project N ${ }^{\circ}$ 644013) and the Generalitat de Catalunya (project ref. 2014-SGR-130). E. Roitero acknowledges the Erasmus Mundus Doctoral Programme DocMASE of the European Commission (FPA 2011-0020). F. Mücklich wishes to acknowledge the EFRE Funds of the European Commission for support of activities within the AME-Lab project. The authors would also like to thank Dr. F. G. Marro for the setup of the B3B test and Dr. T. Trifonov for his help during SEM sessions.

\section{References}

[1] Implants for surgery - Ceramic materials based on yttria-stabilized tetragonal zirconia (Y-TZP) - EN ISO 13356:2013, (2013). 
[2] I. Denry, J. Kelly, State of the art of zirconia for dental applications., Dent. Mater. 24 (2008) 299-307. doi:10.1016/j.dental.2007.05.007.

[3] J. Chevalier, L. Gremillard, Ceramics for medical applications: A picture for the next 20 years, J. Eur. Ceram. Soc. 29 (2009) 1245-1255. doi:10.1016/j.jeurceramsoc.2008.08.025.

[4] Y. Ito, Surface micropatterning to regulate cell functions., Biomaterials. 20 (1999) 233342. http://www.ncbi.nlm.nih.gov/pubmed/10614939.

[5] M.G. Holthaus, S. Twardy, J. Stolle, O. Riemer, L. Treccani, E. Brinksmeier, K. Rezwan, Micromachining of ceramic surfaces: Hydroxyapatite and zirconia, J. Mater. Process. Technol. 212 (2012) 614-624. doi:10.1016/j.jmatprotec.2011.06.007.

[6] A.N. Samant, N.B. Dahotre, Laser machining of structural ceramics-A review, J. Eur. Ceram. Soc. 29 (2009) 969-993. doi:10.1016/j.jeurceramsoc.2008.11.010.

[7] A. Kurella, N.B. Dahotre, Review paper: surface modification for bioimplants: the role of laser surface engineering., J. Biomater. Appl. 20 (2005) 5-50. doi:10.1177/0885328205052974.

[8] F. Mücklich, a. Lasagni, C. Daniel, Laser interference metallurgy - Periodic surface patterning and formation of intermetallics, Intermetallics. 13 (2005) 437-442. doi:10.1016/j.intermet.2004.07.005.

[9] E. Roitero, F. Lasserre, M. Anglada, F. Mücklich, E. Jiménez-Piqué, A parametric study of laser interference surface patterning of dental zirconia: Effects of laser parameters on topography and surface quality, Dent. Mater. (2016) 10-14. doi:10.1016/j.dental.2016.09.040.

[10] W.D. Kingery, H.K. Bowen, D.R. Uhlmann, Introduction to ceramics, second, 1976.

[11] E. Roitero, F. Lasserre, J.J. Roa, M. Anglada, F. Mücklich, E. Jiménez-Piqué, Nanosecondlaser patterning of 3Y-TZP: Damage and microstructural changes, J. Eur. Ceram. Soc. 37 (2017) 4876-4887. doi:10.1016/j.jeurceramsoc.2017.05.052.

[12] E. Roitero, M. Ochoa, M. Anglada, F. Mücklich, E. Jiménez-Piqué, Low temperature degradation of laser patterned 3Y-TZP: Enhancement of resistance after thermal treatment, J. Eur. Ceram. Soc. in press (2017) 0-1. doi:10.1016/j.jeurceramsoc.2017.10.044.

[13] R. Danzer, A general strength distribution function for brittle materials, J. Eur. Ceram. Soc. 10 (1992) 461-472. doi:10.1016/0955-2219(92)90021-5.

[14] C. Daniel, J. Drummond, R.A. Giordano, Improving Flexural Strength of Dental Restorative Ceramics Using Laser Interference Direct Structuring, J. Am. Ceram. Soc. 91 (2008) 3455-3457. doi:10.1111/j.1551-2916.2008.02642.x.

[15] P.P. Shukla, J. Lawrence, Characterization and compositional study of a ZrO2 engineering ceramic irradiated with a fibre laser beam, Opt. Laser Technol. 43 (2011) 1292-1300. doi:10.1016/j.optlastec.2011.03.026. 
[16] B.S. Yilbas, Laser treatment of zirconia surface for improved surface hydrophobicity, J. Alloys Compd. 625 (2015) 208-215. doi:10.1016/j.jallcom.2014.11.069.

[17] C. Daniel, F. Mücklich, Z. Liu, Periodical micro-nano-structuring of metallic surfaces by interfering laser beams, Appl. Surf. Sci. 208-209 (2003) 317-321. doi:10.1016/S01694332(02)01381-8.

[18] M. Hans, C. Gachot, F. Müller, F. Mücklich, Direct laser interference structuring as a tool to gradually tune the wetting response of titanium and polyimide surfaces, Adv. Eng. Mater. 11 (2009) 795-800. doi:10.1002/adem.200900115.

[19] R. Danzer, W. Harrer, P. Supancic, T. Lube, Z. Wang, A. B??rger, The ball on three balls test-Strength and failure analysis of different materials, J. Eur. Ceram. Soc. 27 (2007) 1481-1485. doi:10.1016/j.jeurceramsoc.2006.05.034.

[20] A. Börger, P. Supancic, R. Danzer, The ball on three balls test for strength testing of brittle discs: stress distribution in the disc, J. Eur. Ceram. Soc. 22 (2002) 1425-1436. doi:10.1016/S0955-2219(01)00458-7.

[21] W. Weibull, A statistical distribution function of wide applicability, J. Appl. Mech. 18 (1951) 293-297. doi:citeulike-article-id:8491543.

[22] D. Munz, T. Fett, Ceramics : mechanical properties, failure behaviour, materials selection, Springer, 1999. doi:10.1007/978-3-642-33848-9.

[23] W.C. Oliver, G.M. Pharr, Measurement of hardness and elastic modulus by instrumented indentation: Advances in understanding and refinements to methodology, J. Mater. Res. 19 (2004) 3-20. doi:10.1557/jmr.2004.19.1.3.

[24] Y. Gaillard, M. Anglada, E. Jiménez-Piqué, Nanoindentation of yttria-doped zirconia: Effect of crystallographic structure on deformation mechanisms, J. Mater. Res. 24 (2009) 719-727. doi:10.1557/jmr.2009.0091.

[25] Y. Gaillard, E. Jiménez-Piqué, F. Soldera, F. Mücklich, M. Anglada, Quantification of hydrothermal degradation in zirconia by nanoindentation, Acta Mater. 56 (2008) 42064216. doi:10.1016/j.actamat.2008.04.050.

[26] A.C. Fischer-Cripps, A review of analysis methods for sub-micron indentation testing $₫$, Vacuum. 58 (2000) 569-585.

[27] R. Danzer, On the relationship between ceramic strength and the requirements for mechanical design, J. Eur. Ceram. Soc. 34 (2014) 3435-3460.

doi:10.1016/j.jeurceramsoc.2014.04.026.

[28] M. Turon-Vinas, M. Anglada, Fracture toughness of zirconia from a shallow notch produced by ultra-short pulsed laser ablation, J. Eur. Ceram. Soc. 34 (2014) 3865-3870. doi:10.1016/j.jeurceramsoc.2014.05.009.

[29] K. Noguchi, M. Fujita, T. Masaki, M. Mizushina, Tensile Strength of Yttria-Stabilized Tetragonal Zirconia Polycrystals, J. Am. Ceram. Soc. 72 (1989) 1305-1307. doi:10.1111/j.1151-2916.1989.tb09736.x. 
[30] S.G. Demos, M. Staggs, M.R. Kozlowski, Investigation of processes leading to damage growth in optical materials for large-aperture lasers., Appl. Opt. 41 (2002) 3628-3633. doi:10.1364/AO.41.003628.

[31] H. Bercegol, P. Grua, D. Hebert, J.P. Morreeuw, Progress in the understanding of fracture related laser damage of fused silica - art. no. 672003, Laser-Induced Damage Opt. Mater. 2007. 6720 (2008) 72003. doi:10.1117/12.752830.

[32] W.M. Steen, LASER MATERIAL PROCESSING, third edit, n.d.

[33] L. Ceseracciu, M. Anglada, E. Jiménez-Piqué, Hertzian cone crack propagation on polycrystalline materials: Role of R-curve and residual stresses, Acta Mater. 56 (2008) 265-273. doi:10.1016/j.actamat.2007.09.030. 